\title{
Successful Translation of Fluorescence Navigation During Oncologic Surgery: A Consensus Report
}

\author{
Eben L. Rosenthal ${ }^{1}$, Jason M. Warram²,3, Esther de Boer ${ }^{2,4}$, James P. Basilion ${ }^{5}$, Merrill A. Biel ${ }^{6}$, Matthew Bogyo ${ }^{7,8}$, \\ Michael Bouvet $^{9}$, Brian E. Brigman ${ }^{10}$, Yolonda L. Colson ${ }^{11}$, Steven R. DeMeester ${ }^{12}$, Geoffrey C. Gurtner ${ }^{13}$, \\ Takeaki Ishizawa ${ }^{14}$, Paula M. Jacobs ${ }^{15}$, Stijn Keereweer ${ }^{16}$, Joseph C. Liao ${ }^{17}$, Quyen T. Nguyen ${ }^{18}$, James M. Olson ${ }^{19,20}$, \\ Keith D. Paulsen ${ }^{21}$, Dwaine Rieves ${ }^{22}$, Baran D. Sumer ${ }^{23}$, Michael F. Tweedle ${ }^{24}$, Alexander L. Vahrmeijer ${ }^{25}$, \\ Jamey P. Weichert ${ }^{26}$, Brian C. Wilson ${ }^{27,28}$, Michael R. Zenn ${ }^{29}$, Kurt R. Zinn ${ }^{3}$, and Gooitzen M. van Dam ${ }^{30}$
}

${ }^{1}$ Department of Otolaryngology, Stanford University, Stanford, California; ${ }^{2}$ Division of Otolaryngology, Department of Otolaryngology, University of Alabama at Birmingham, Birmingham, Alabama; ${ }^{3}$ Department of Radiology, University of Alabama at Birmingham, Birmingham, Alabama; ${ }^{4}$ Department of Surgery, University Medical Center Groningen, University of Groningen, Groningen, The Netherlands; ${ }^{5}$ Case Center for Imaging Research, NFCR Center for Molecular Imaging, Department of Radiology Case Western Reserve University, Cleveland, Ohio; ${ }^{6}$ Department of Otolaryngology-Head and Neck Surgery, University of Minnesota, Minneapolis, Minnesota; ${ }^{7}$ Department of Pathology, Stanford School of Medicine, Stanford, California; ${ }^{8}$ Department of Cancer Biology Program, Stanford School of Medicine, Stanford, California; ${ }^{9}$ Department of Surgery, University of California San Diego, La Jolla, California; ${ }^{10}$ Division of Orthopaedic Surgery, Department of Surgery, Duke University Medical Center, Durham, North Carolina; ${ }^{11}$ Division of Thoracic Surgery, Brigham and Women's Hospital, Boston, Massachusetts; ${ }^{12}$ Department of Surgery, Keck School of Medicine of the University of Southern California, Los Angeles, California; ${ }^{13}$ Hagey Laboratory for Pediatric Regenerative Medicine, Division of Plastic and Reconstructive Surgery, Department of Surgery, Stanford University School of Medicine, Stanford, California; ${ }^{14}$ Artificial Organ and Transplantation Surgery Division, Department of Surgery, Graduate School of Medicine, University of Tokyo, Tokyo, Japan; ${ }^{15}$ Cancer Imaging Program, DCTD, NCI, Bethesda, Maryland; ${ }^{16}$ Department of Otorhinolaryngology Head and Neck Surgery, Erasmus Medical Center, Rotterdam, The Netherlands; ${ }^{17}$ Department of Urology and Bio-X Program, Stanford University School of Medicine, Stanford, California; ${ }^{18}$ Division of Head and Neck Surgery, University of California San Diego, San Diego, California; ${ }^{19}$ Clinical Research Division, Fred Hutchinson Cancer Research Center, Seattle, Washington; ${ }^{20}$ Seattle Children's Hospital \& Regional Medical Center, Seattle, Washington; ${ }^{21}$ Department of Diagnostic Radiology, Geisel School of Medicine, Dartmouth College, Hanover, New Hampshire; ${ }^{22}$ NDA Partners LLC, Washington, District of Columbia; ${ }^{23}$ Department of Otolaryngology, Simmons Comprehensive Cancer Center, University of Texas Southwestern Medical Center, Dallas, Texas; ${ }^{24}$ Department of Radiology, The Wright Center for Innovation in Biomedical Imaging, The Ohio State University, Columbus, Ohio; ${ }^{25}$ Department of Surgery, Leiden, University Medical Center, Leiden, The Netherlands; ${ }^{26}$ Department of Radiology, University of Wisconsin, Madison, Wisconsin; ${ }^{27}$ Princess Margaret Cancer Centre, UHN, Toronto, Ontario, Canada; ${ }^{28}$ Medical Biophysics, University of Toronto, Toronto, Ontario, Canada; ${ }^{29}$ Plastic and Reconstructive Surgery, Duke University Medical Center, Durham, North Carolina; and ${ }^{30}$ Department of Surgery, Nuclear Medicine and Molecular Imaging and Intensive Care, University Medical Center Groningen, University of Groningen, The Netherlands, on behalf of the International Society of Image Guided Surgery (ISIGS)

Navigation with fluorescence guidance has emerged in the last decade as a promising strategy to improve the efficacy of oncologic surgery. To achieve routine clinical use, the onus is on the surgical community to objectively assess the value of this technique. This assessment may facilitate both Food and Drug Administration approval of new optical imaging agents and reimbursement for the imaging procedures. It is critical to characterize fluorescence-guided procedural benefits over existing practices and to elucidate both the costs and the safety risks. This report is the result of a meeting of the International Society of Image Guided Surgery (www.isigs.org) on February 6, 2015, in Miami, Florida, and reflects a consensus of the participants' opinions. Our objective was to critically evaluate the imaging platform technology and optical imaging agents and to make recommendations for successful clinical trial development of this highly promising approach in oncologic surgery.

Received Apr. 6, 2015; revision accepted Sep. 24, 2015.

For correspondence or reprints contact: Eben L. Rosenthal, Stanford Cancer Center, 875 Blake Wilbur Dr., Stanford, CA 94305.

E-mail: elr@stanford.edu

Published online Oct. 8, 2015

COPYRIGHT (C) 2016 by the Society of Nuclear Medicine and Molecular Imaging, Inc.
Key Words: fluorescence-guided surgery; consensus report; regulatory guidance; ISIGS

J Nucl Med 2016; 57:144-150

DOI: 10.2967/jnumed.115.158915

A lthough the field of surgery has recently experienced tremendous advances in optical technologies and robotics, one area that has remained constant is the dependence on visual and palpable cues that differentiate diseased from healthy tissue. Reliance on white light limits the visual contrast available to the operating surgeon to a narrow dynamic range in the colorimetric spectrum. Consequently, the ability to identify subclinical and deep-seated disease states during oncologic surgery is difficult, and the surgeon must rely on nonspecific visual changes and manual palpation of subtle irregularities to guide cancer excision. The most common method of intraoperative margin control is frozen section analysis; however, this technique is time-intensive and can sample only a small fraction of the wound bed, resulting in reversal in up to $19 \%$ of cancer cases 
$(1,2)$. Given that the primary treatment modality for most solid tumors is radical surgery, and because positive margins (defined as tumor cells present at the cut edge of the surgical specimen) are associated with increased local recurrence and poor prognoses, real-time intraoperative distinction between tumor and normal tissue is urgently needed to improve surgical outcomes, and to simultaneously prevent under- and overtreatment.

Conventional anatomic imaging modalities, such as MR imaging, ultrasound, and CT, have been adopted for use in the operating room. Additionally, advanced imaging suites with combined operating rooms are becoming more commonplace in major institutions. Many of these include intraoperative PET imaging to be used in adjunct with MR imaging for tumor mapping and validating tumor excision completeness during surgery. Unfortunately, these imaging techniques are neither real-time nor tumor-specific. Furthermore, conventional imaging modalities are typically quite costly and cannot be applied easily in the surgical field of view. Use of optical imaging for cancer-specific navigation has been successfully introduced in glioma surgery with the fluorescent agent 5-aminolevulinic acid (5-ALA) $(3,4)$. Additional clinical trials evaluating fluorescence-guided techniques are ongoing (Table 1). Evaluation of cetuximab-IRDye800 in head and neck cancer has suggested that fluorescently labeled antibodies are safe and can detect subclinical fragments of tumor. To extend optical imaging techniques to other oncologic settings, future clinical trials of image-guided surgery will need to be designed in a way that rigorously evaluates the added benefit for patients, while also assessing the cost-effectiveness. Unlike the introduction of a new drug for the treatment of cancer, surgical trials evaluating fluorescence-guided resection present unique hurdles, such as limited surgical procedure standardization, difficulty in patient randomization, what endpoints to use as assessment metrics, and variations in how surgeons currently determine the normal versus tumor interface during surgery. Therefore, it is critical for the surgical community to address these issues early in the clinical development of optical imaging agents and technology, particularly considering the limited commercial resources available for developing these products.

To cooperatively accomplish these goals, the International Society of Image Guided Surgery was formed, and a consensus meeting was held in February 2015 to discuss regulatory pathways, clinical trial design, and patient safety. Attendees included an international assembly of surgeons, scientists, and regulatory administrators who cooperatively addressed specific issues facing the translation of this technology. The objective of this meeting was to identify optimal routes for imaging agent and device regulatory approval and successful clinical trial outcomes. This consensus of the meeting attendees may serve as standardized guidance for navigating the regulatory process and designing successful clinical trials in fluorescence-guided surgery for oncologic resection. A summary of recommendations is given in Table 2 .

\section{FOOD AND DRUG ADMINISTRATION (FDA) REGULATORY PATHWAYS AND OBTAINING AN INVESTIGATIONAL NEW DRUG (IND)}

Early-phase clinical trials will need to establish the safety of optical imaging contrast agents as well as that of the accompanying imaging device, if not already approved. Trials designed to evaluate the safety of imaging agents typically evaluate small, single doses of the agent, which may have limited potential for probe-induced toxicity. Still, early-phase clinical studies are essential to detect early safety events. However, trials with limited numbers of patients often have a more difficult time detecting infusion reactions or other immunologic events that tend to be rare and dose-independent. Conventional phase I study designs often use dose escalation to determine a maximally tolerated dose for therapeutic drugs. Maximally tolerated doses are not appropriate for imaging agents because optimal imaging contrast should be achieved well below toxic dose $(5-8)$. When disease-specific contrast is the objective, reducing the uptake in normal tissues is just as critical as increasing the disease-specific uptake. Therefore, imaging agent dosing should be scaled to determine optimal delineation of cancer compared with normal tissue, and the optimal contrast may not necessarily correlate with increasing dose. Nevertheless, in comparison to radionuclide imaging drugs, which emit high-energy photons with little tissue attenuation, the signals from optical imaging agents are subject to greater attenuation and may require greater doses to achieve suitable contrast. Considering these realities, the consensus was that a dose-escalation study designed to detect optimal imaging contrast (optimal tumor-to-background ratio) during surgery as well as safety is generally preferred over a trial design or dosing schedule intended solely to assess imaging agent safety. Furthermore, early-phase clinical trials should be designed to identify the appropriate timing of imaging agent administration relative to surgery.

\section{Exploratory IND (eIND) or Microdosing}

In 2006, to reduce the significant demand of resources and time required for traditional IND applications, the FDA published a guidance document that described the eIND. The eIND refers to a regulatory process that allows very early clinical trials (phase 0 ) to proceed with relatively limited clinical data if the investigational imaging agent is administered at a subpharmaceutical dose $(\leq 100$ micrograms of a synthetic drug or $\leq 30 \mathrm{nmol}$ of a protein) $(9,10)$. The primary reason to obtain an eIND, as opposed to the traditional IND process, is to accelerate first-in-human experience and to obtain proof of concept and pharmacokinetic data early in development. If the eIND study is successful, then additional toxicology will need to be performed for submission of a traditional IND application. Therefore, full imaging drug development proceeds only under the traditional IND pathway. Trial designs for eIND studies must involve limited human exposure (generally a single drug administration) and have no goal of producing therapeutic or diagnostic benefits. Depending on the affinity for the target and the clearance characteristics for an optical imaging probe, this approach may or may not be useful for optical probes. Guidance documents about obtaining an eIND can be found at the FDA website (11).

\section{IND-Enabling Toxicology}

Filing a traditional IND requires submission of sufficient nonclinical toxicology and manufacturing information to support the safety of the proposed clinical trial. The process of compiling an IND for a new imaging agent is well beyond the scope of this document; however, there are several aspects to the development of a cancer-specific fluorescent imaging agent that are worth noting. Nonclinical toxicology studies should be designed around general pharmacology (mechanism of action and dose-related effects), pharmacokinetics, pharmacodynamics, safety, assessment of the major organs vulnerable to toxicity, and an estimate of the margin of safety between a clinical and a toxic dose. The most conservative approach in first-in-human trials using a dose-escalation strategy usually entails beginning at the microdose level. To obtain these data, doses in nonclinical studies should bracket the expected clinical dose, including a dose that is significantly higher than the anticipated highest dose and usually requires exploration of at 
TABLE 1

Ongoing Clinical Trials

\begin{tabular}{|c|c|c|c|c|c|c|}
\hline Biologic agent & Trial phase & Identifier & Condition & Target & Principal investigator & Sponsor \\
\hline $\begin{array}{l}\text { Bevacizumab- } \\
\text { IRDye800CW }\end{array}$ & I & NCT01691391 & FAP & VEGF & W.B. Nagengast & $\begin{array}{l}\text { University Medical } \\
\text { Centre Groningen }\end{array}$ \\
\hline $\begin{array}{l}\text { Bevacizumab- } \\
\text { IRDye800CW }\end{array}$ & I & NCT01972373 & Rectal cancer & VEGF & W.B. Nagengast & $\begin{array}{l}\text { University Medical } \\
\text { Centre Groningen }\end{array}$ \\
\hline $\begin{array}{l}\text { Bevacizumab- } \\
\text { IRDye800CW }\end{array}$ & 1 & NCT01508572 & $\mathrm{BC}$ & VEGF & G.M. van Dam & $\begin{array}{l}\text { University Medical } \\
\text { Centre Groningen }\end{array}$ \\
\hline $\begin{array}{l}\text { Bevacizumab- } \\
\text { IRDye800CW }\end{array}$ & I & NCT02129933 & $\begin{array}{l}\text { Premalignant } \\
\text { esophageal } \\
\text { lesions }\end{array}$ & VEGF & W.B. Nagengast & $\begin{array}{l}\text { University Medical } \\
\text { Centre Groningen }\end{array}$ \\
\hline $\begin{array}{l}\text { Cetuximab- } \\
\text { IRDye800CW }\end{array}$ & I & NCT01987375 & HNC & EGFR & E.L. Rosenthal & UAB \\
\hline $\begin{array}{l}\text { Panitumumab- } \\
\text { IRDye800CW }\end{array}$ & $\mathrm{P}$ & NCT01998273 & $\mathrm{HNC}$ & EGFR & E.L. Rosenthal & UAB \\
\hline MDX1201-A488 & $\mathrm{P}$ & NCT02048150 & $\mathrm{PCa}$ & PSMA & T. Wilson & $\begin{array}{l}\text { City of Hope Medical } \\
\text { Center }\end{array}$ \\
\hline ProstaFluor & $\mathrm{O}$ & NCT01173146 & $\mathrm{PCa}$ & PSMA & D. Herrell & Spectros Corp. \\
\hline AVB-620 & I & NCT02391194 & $\mathrm{BC}$ & Proteases & A. Wallace & Avelas Biosciences \\
\hline \multirow[t]{2}{*}{ BLZ-100 } & I & NCT02234297 & $\begin{array}{l}\text { Glioma brain } \\
\text { tumors }\end{array}$ & MMP-2 & C.G. Patil & Blaze Bioscience Inc. \\
\hline & & & & Annexin VII & & \\
\hline \multirow[t]{2}{*}{ 5-ALA } & I & NCT00870779 & $\begin{array}{l}\text { HGG, } \\
\text { meningioma, } \\
\text { pituitary } \\
\text { adenoma, or } \\
\text { metastasis }\end{array}$ & Porphyrin & D.W. Roberts & $\begin{array}{l}\text { Dartmouth-Hitchcock } \\
\text { Medical Center }\end{array}$ \\
\hline & & & & Synthesis & K. Paulsen & \\
\hline \multirow[t]{2}{*}{ 5-ALA } & II & NCT01445691 & Brain tumors & Porphyrin & C. Hadjipanayis & Emory University \\
\hline & & & & Synthesis & & \\
\hline \multirow[t]{2}{*}{ 5-ALA } & I & NCT01128218 & Brain tumors & Porphyrin & J.W. Cozzens & $\begin{array}{l}\text { Southern Illinois } \\
\text { University }\end{array}$ \\
\hline & II & & & Synthesis & & \\
\hline \multirow[t]{2}{*}{ 5-ALA } & $\mathrm{O}$ & NCT01837225 & $\mathrm{BC}$ & Porphyrin & W.L. Leong & UHN, Toronto \\
\hline & & & & Synthesis & R.S. DaCosta & \\
\hline \multirow[t]{2}{*}{ 5-ALA } & II & NCT00752323 & Brain tumors & Porphyrin & A. Sloan & Case CCC \\
\hline & & & & Synthesis & & \\
\hline \multirow[t]{2}{*}{ 5-ALA } & I & NCT02191488 & Brain tumors & Porphyrin & D.W. Roberts & $\begin{array}{l}\text { Dartmouth-Hitchcock } \\
\text { Medical Center }\end{array}$ \\
\hline & & & & Synthesis & & \\
\hline \multirow[t]{2}{*}{ 5-ALA } & III & NCT01502280 & Glioblastoma & Porphyrin & N. Sanai & $\begin{array}{l}\text { St. Joseph's Hospital } \\
\text { and Medical } \\
\text { Center }\end{array}$ \\
\hline & & & & Synthesis & & \\
\hline \multirow[t]{2}{*}{ 5-ALA } & $\mathrm{O}$ & NCT02155452 & Glioblastoma & Porphyrin & A.V. Moiyadi & $\begin{array}{c}\text { Tata Memorial } \\
\text { Hospital }\end{array}$ \\
\hline & & & & Synthesis & & \\
\hline LUM015 & I & NCT01626066 & Sarcoma, BC & Cathepsin & D. Kirsch & $\begin{array}{l}\text { Duke University } \\
\text { Medical Center }\end{array}$ \\
\hline
\end{tabular}

$\mathrm{FAP}=$ familial adenomatous polyposis; VEGF = vascular endothelial growth factor; $\mathrm{BC}=$ breast cancer; HNC $=$ head and neck cancer; EGFR = epidermal growth factor receptor; UAB = University of Alabama at Birmingham; $\mathrm{P}=$ pilot; $\mathrm{PCa}=$ prostate cancer; $\mathrm{O}=$ observational; $\mathrm{HGG}=$ high-grade glioma; UHN = University Health Network; CCC = Comprehensive Cancer Center. Registered ongoing clinical trials as of July 16, 2015. 
least 3 drug dosages (or possibly a single high dose). Generally a rodent and a nonrodent species are selected for these studies, but the test species must be pharmacologically responsive or have the appropriate antigen specificity to the proposed study drug. Agents with unique toxicities that may be less dose-related in humans, such as certain proteins (allergic response) or novel receptor targeting agents, may require use of nonhuman primates. The manufacturing processes and formulation of the study drug for nonclinical toxicology does not need to meet the standards of those for phase 3 clinical studies; however, the drug formulation should be similar enough to ensure that the toxicology results can support full product development, including phase 3 trials. The FDA imaging guidance documents are found at the FDA website (12).

The FDA pre-IND consultation program provides a unique opportunity to obtain imaging agent-specific advice regarding the data and information to submit with an IND application. A productive pre-IND meeting requires that the IND sponsor have a nonclinical toxicology, manufacturing, and clinical protocol plan in place so the FDA can answer specific questions about the suitability of the strategy, such as "Does the design of the extended follow-up, single-dose rodent study appear reasonable to support the proposed clinical trial doses of the imaging agent?" Traditionally the FDA will not provide input to open-ended questions such as "What evaluations should be performed during the toxicology experiments?" Current FDA guidance documents provide much in the way of nonclinical, manufacturing, and clinical trial design advice, and the FDA expects sponsors (the individual or organization responsible for actually submitting the IND) to have incorporated advice from these documents in the development of their IND submission plans. Therefore, a relatively mature imaging agent/technology development plan is critical to provide the greatest benefit to the sponsor. A pre-IND meeting may be conducted over the telephone or in person; however, the consensus is that in-person meetings generally facilitate discussion of critical topics.

Fluorescent labeling of approved drugs or biologic products, such as therapeutic antibodies, have been be translated to the clinic with fewer toxicology studies than new optical imaging agents $(13,14)$. The purpose of the toxicology studies in this setting is to demonstrate that the fluorescently labeled agent has the same toxicity and pharmacokinetic profile as the unlabeled, approved product. On the basis of limited results, when fluorescently labeling the approved product, a low dye-to-product mass ratio (15) favors similar clearance rates and limited change in the toxicity/antigenicity of the molecule. Although the intellectual property issues surrounding these fluorescently labeled products remain complex, successful clinical translation may be more efficient in comparison to unique imaging agents, rather than for agents with no prior extensive clinical testing. Once an IND has been successfully opened, sponsors may submit amendments to the IND to support the enrollment of additional patients to the ongoing clinical protocol or new protocols investigating the investigational optical

TABLE 2

Summary of Recommendations

\begin{tabular}{|c|c|}
\hline Recommendation & Summary \\
\hline 1 & $\begin{array}{l}\text { - Microdosing can be used to confirm target specificity but is insufficient for intraoperative } \\
\text { imaging. }\end{array}$ \\
\hline 2 & $\begin{array}{l}\text { - It is currently unclear if the device and the drug product should be paired or general } \\
\text { parameters for devices set for each drug product. Discussions during a pre-IND meeting } \\
\text { or during a subsequent meeting with FDA (such an end of phase } 2 \text { meeting) should } \\
\text { address combination product development considerations. However, it is preferable for } \\
\text { device manufacturers to seek marketing approval without restriction of the device to any } \\
\text { specific optical imaging agent if the device can successfully image more than } 1 \\
\text { fluorophore within the device's excitation/emission spectrum. }\end{array}$ \\
\hline 3 & $\begin{array}{l}\text { Dose- and time-ranging studies should be performed in phase I clinical trial setting. } \\
\text { A dose-escalation study designed to detect optimal imaging contrast during surgery as } \\
\text { well as safety is generally preferred over a trial design or dosing schedule intended solely } \\
\text { to assess imaging agent safety. }\end{array}$ \\
\hline 4 & $\begin{array}{l}\text { - Considering the multiple indications optical imaging agents may ultimately be approved } \\
\text { for, the common verification for a cancer indication should be demonstration that the } \\
\text { imaging successfully delineates normal from abnormal tissue. There is a consensus that a } \\
\text { standard methodology should be introduced to accomplish the correlation of } \\
\text { fluorescence with the presence of tumor. }\end{array}$ \\
\hline 5 & $\begin{array}{l}\text { - The general consensus is that in order for optically guided surgery to advance to routine } \\
\text { clinical use, there must be a widely adopted methodology for fluorescence assessment. } \\
\text { This degree of standardized and objective assessment will be helpful for regulatory } \\
\text { approval to critically demonstrate the ability of the technique to provide disease-specific } \\
\text { contrast. }\end{array}$ \\
\hline 6 & $\begin{array}{l}\text { - Acceptable toxicity for optical contrast agents for oncologic surgery should be between } \\
\text { diagnostic and therapeutic agents. }\end{array}$ \\
\hline 7 & $\begin{array}{l}\text { - Grade } 2 \text { toxicity in } 20 \% \text { of the population is an acceptable threshold as a dose limiting } \\
\text { toxicity. }\end{array}$ \\
\hline
\end{tabular}


imaging agent in other cancer types. As with the initial clinical protocol development, these alterations in the clinical development program must maintain compliance with the local Institutional Review Board expectations.

\section{Pairing of Imaging Device and Agent}

Before the marketing of new imaging devices, the FDA requires that the manufacturer (typically the sponsor) obtain clearance for marketing the device under a $510(\mathrm{k})$ clearance pathway or approval under the PreMarket Approval process (16). The 510(k) premarket submission mechanism allows an FDA review and clearance for marketing based on the substantial equivalence of a new device to an already FDA-cleared or -approved device (17). Before the submission of a $510(\mathrm{k})$ application or PreMarket Approval process to support marketing of a new device, the regulatory development of the device proceeds in a manner similar to that for a new optical imaging agent. That is, clinical trials often evaluate the new imaging device after submission of an investigational device exemption application to the FDA. If the new device is paired with a new optical imaging agent in the initial clinical trial, the FDA often requests submission of supporting safety information for both the device and the imaging agent with an IND, rather than submission of an IND as well as an investigational device exemption application. This agent-device IND submission process is one of the topics to consider for a pre-IND meeting.

The pairing of a fluorescence contrast agent and imaging device for regulatory approval as combination products remains polarizing. If an imaging agent is intended to be imaged using a specific device, then FDA classifies the agent and device as a combination product. To expand the range of devices and potential imaging agents that could be used interchangeably in the future, the general consensus from this meeting was that it is preferable for device manufacturers to seek marketing approval without restriction of the device to any specific optical imaging agent if the device can successfully image more than 1 fluorophore within the device's excitation/emission spectrum. Discussions during a pre-IND meeting or during a subsequent meeting with FDA (such as an end of phase 2 meeting) should address combination product development considerations. The device manufacturer and optical imaging agent sponsor should develop a drug-device development program in preparation for this meeting. Fluorescent agents could be paired with devices based on a range of wavelength overlap, rather than pair specific devices with specific probes.

Rather than using an investigational imaging device in a clinical trial, using an FDA-cleared or -approved imaging device with an established installation base can expedite imaging agent development. However, this approach may not be feasible if the device is not designed specifically for the imaging spectrum of the fluorophore. Furthermore, repurposed devices may not be ideal for certain intraoperative surgical indications due to poor ergonomics, size limitations, and constrained integrated software. In general, imaging devices themselves are considered low risk or nonsignificant risk; however, advanced-stage clinical trials will have to address how the information obtained from the fluorophore/device combination will be used in clinical decision making.

\section{CLINICAL TRIAL DESIGN}

\section{Early-Phase Clinical Endpoints}

The focus of phase I clinical trials should be on safety of the imaging agent and device. Secondary endpoints would include identification of the appropriate agent dose and timing for surgical intervention. Optimal imaging agent dose should be defined by consideration of anticipated agent toxicities (if any), tumor-tobackground ratio required to differentiate diseased from normal tissue, patient work flow, and economics. Ultimately, the optimal dose and timing of the imaging agent will be target- and clearancedependent rather than dependent on the fluorescent probe. These parameters are highly reliant on the pharmacokinetics of the agent and the physiology of the target. For example, extracellular matrix targets in the stromal compartment can exhibit contrasting characteristics to targets that are membrane-bound. Additionally, the optimal dose and timing can depend on half-life and targeting of the agent; for example, the imaging timing for an antibody may markedly differ from that of an antibody fragment or Affibody molecules. If these optical imaging agents are to be approved for the routine management of oncologic surgery, it will be necessary to demonstrate that fluorescent signal correlates with the presence of cancer. Eventually there will be a need to demonstrate patient benefit and clinical usefulness, but the cost and long-term follow-up to demonstrate these endpoints are often difficult to implement in early-stage clinical trials. Safety and efficacy may be all that is required for IND approval, but proof of patient outcomes and economics are increasingly required by payers and necessary for widespread reimbursement and use of a new agent.

\section{Acceptable Toxicity}

Diagnostic imaging agents are often held to a high standard for safety because they are commonly given to a vast number of patients, sometimes at regular intervals, for a range of disease types, including some with benign outcomes. Because of these factors, federal regulation considers anything over a grade 1 reaction, defined as mild or asymptomatic response, unacceptable for imaging agents. In chemotherapeutic trials with cytotoxic agents, an acceptable toxicity is usually a grade 1 or 2 reaction related to the study drug, albeit some grade 3 reactions can occur. Because the proposed use of optical contrast for surgical resection of cancer spans the gap between diagnostic and therapeutic imaging, there should be unique considerations granted for intraoperative imaging agents considering the disease is usually life-threatening and the patient is undergoing a major procedure, usually under general anesthesia. Depending on the nature of the disease type, the surgical intervention, and prognosis, the group considered that a limited number of grade 2 adverse events, defined as moderate with local or noninvasive intervention indicated, would be acceptable. This decision applies primarily to tumors requiring aggressive surgical intervention that have a significantly high risk of mortality, compared with procedures for which there are limited risks associated with the intervention. Patients should be followed for a sufficient duration of time to thoroughly evaluate the potential toxicity of the imaging agent/device/procedure; at a minimum, the follow-up duration should extend to at least 4 times the half-life of the imaging agent.

\section{Phase I Trials}

Although early-phase trials should focus on safety and dosing, future trials will be initiated on the basis of data collected during these early examinations. Phase I patients are usually chosen from the likely pool of end-use patients, which is often healthy patients for imaging agents. However, optical surgical navigation agents will be used in patients with biopsy-proven cancers undergoing surgery, so the pool for phase I trials will be patients already triaged to surgery, allowing inclusion of secondary imaging endpoints in the results. Apart from safety data, the success of the imaging strategy (agent and device) can be evaluated in several ways. First, sensitivity 
and specificity of the intraoperative imaging strategy can be calculated relative to surgeon assessment and pathologic assessment. For example, perform the surgery in the absence of fluorescenceguidance then apply the imaging postresection to assess specimens and surgical beds. Second, the tumor-to-background ratio will provide information on the ability of the imaging strategy to provide sufficient contrast for disease delineation. Third, the specificity of the agent and device to accurately demarcate the disease border can be measured by mapping the fluorescence edge with the pathologic disease margin. In cases in which a stable fluorescent imaging agent is used, the agent will survive pathologic processing, and fluorescence microscopy can be used to correlate fluorescence with histologic evidence of tumor on hematoxylin and eosin-stained sections. Furthermore, immunohistochemistry can be performed to map specific tumor antigens with fluorescence intensity to determine successful drug penetration and targeting.

For an indication of structure delineation, FDA guidance documents define clinical usefulness as the ability to distinguish normal from abnormal tissue. Therefore, the most critical endpoint is demonstrating that the presence of fluorescence is specific for cancer. Correlating the fluorescence during surgical imaging presents a greater challenge due to the detailed mapping that is required to trace the fluorescent signal through formalin fixation and histologic processing. Considering the multiple indications optical imaging agents may ultimately be approved for, the common verification required for a cancer indication is demonstration that the imaging successfully delineates normal from abnormal tissue. There is a consensus that a standard methodology should be introduced to accomplish the correlation of fluorescence with the presence of tumor.

When discussing this technology, it is often asked "What is the smallest amount of cancer that this technique can detect?" Although detection of subclinical disease remains the primary objective of this imaging strategy, it should be recognized that it is unlikely that this imaging technique will detect a mere few hundred cells. Rather, the goal is to make an incremental improvement on the current ability to detect disease and improve the obvious limitations using intraoperative palpation and visual changes in the tissue. This is especially true for minimally invasive procedures for which there is loss of tactile and 3-dimensional visual feedback. Furthermore, because measurement of fluorescent signal can be confounded by the attenuation of overlying tissue during imaging, the absence of fluorescent signal within a specimen or a wound bed should be interpreted with some caution. Failure to see fluorescence could be explained by the absence of tumor, the presence of residual disease but of insufficient quantity to generate measurable signal, or an optical signal that cannot be measured because of depth of penetration and attenuation. These limitations should be considered when designing early-phase trials to assess novel imaging agents, while simultaneously realizing the full potential in the operating room.

\section{Histology as Gold Standard}

To determine the sensitivity and specificity of the imaging agent, presence of disease must be confirmed with the current gold standard, which is histologic analysis using hematoxylin and eosin staining. However, histopathologic evaluation of tumor specimens is subject to multiple inaccuracies, including sampling error, misinterpretation, and loss of tissue orientation $(1,18,19)$. Correlating histologic evidence of tumor with fluorescence is complicated by these limitations. This particularly applies to false-positives for which the presence of fluorescence within a large tissue mass may not be confirmed by histology because of a failure to fully sample the entire specimen, missing the small region of tumor that was detected by fluorescence. It is not practical to completely evaluate even a small tumor sample (e.g., $1 \times 1 \mathrm{~cm}$ ) by serial sectioning, which would require up to 2,500 slides and thus can be considered impractical and nonexecutable, even in an experimental design. It may be necessary to use polymerase chain reaction-based assays to increase the sensitivity of the gold standard for microscopic disease. Alternatively, trials could be designed to include sectioning based on fluorescence information to further validate the accuracy of the fluorescent signal to detect disease.

\section{Thresholding}

Fluorescence imaging can be threshold-adjusted along a continuum of intensity that must be standardized to an acceptable baseline. The general consensus is that for optically guided surgery to advance to routine clinical use, there must be a widely adopted methodology for fluorescence assessment. For immediate identification of unknown samples in the operating room or pathology for a specific patient, the preferred methodology may be to image the known cancer (tumor mass in situ) and known normal tissue to adjust the threshold to reveal diseased tissue apart from normal. This calibration of threshold would be performed uniquely for each patient. Appropriate thresholding would be performed on the basis of the known samples, revealing the fluorescence intensity of the unknown tissue. This approach is considered optimal, considering a fixed threshold is difficult to establish due to differences in tumor physiology, tissue properties, timing, molecular target expression, and clearance.

For additional standardization, relative quantification may be critical for objective assessment and reporting. Similar to standardized uptake value in assessment and reporting of PET imaging, absolute counts (fluorescence intensity) from unknown tissue and known normal tissue can be used to generate a ratio. A ratiometric threshold for positive disease can be experimentally developed and integrated into the onboard device software to objectively identify disease tissue intraoperatively in real-time using this methodology. A recently published proof-of-concept study demonstrated this approach to be highly reproducible in the fluorescence-guided surgical setting (20). The general consensus is that this degree of standardized and objective assessment will be helpful for regulatory agencies to critically demonstrate the ability of the technique to differentiate normal tissue from disease.

\section{Advanced-Phase Clinical Endpoints}

In many cancer surgeries, positive margins remain a challenge and are associated with poor outcomes. In most cases, the outcome resulting from a single positive surgical margin is not successfully mitigated by subsequent surgery to clear the margin (i.e., reexcision of the positive margin) and will require adjuvant chemotherapy or radiation. But these additional treatments cannot compensate for poor outcomes due to tumor-positive margins. The need for imaging to improve delineation of tumor and normal tissue is an obvious advantage to prevent cutting through cancer, identifying suspicious or close margins, and guiding a consistent margin around the tumor. The value of such an imaging agent would be consistent with how the FDA views approval of such agents. According to the FDA guidance documents for approval of imaging products, section 351(a) of the Public Health Service Act (42 U.S.C. 262(a)): "the ability to locate and outline normal structures or distinguish between normal and abnormal anatomy 
can speak for itself with respect to the clinical value of the information and will not require additional information substantiating clinical usefulness." Centers for Medicare and Medicaid Services and other healthcare payer bodies, however, often have more stringent definitions of efficacy, and often include healthcare economics considerations.

In discussion among oncologic surgeons familiar with this technology, there were several concerns regarding the complexity of alternate clinical trial endpoints to show clinical benefit. Perhaps the most challenging aspect of clinical trial design in this setting is that the standard of care for excision remains surgeon assessment, which is highly variable and subjective-if it looks like cancer, cut it out. An imaging technology that is better than the accepted standard is likely to identify more positive margins than this standard technique when implemented into routine procedures. It should be recognized that both functional outcomes and survival could be overshadowed in studies by postoperative adjuvant therapy, which is commonly performed in cases of positive margins. Furthermore, surgical outcomes are dependent on surgical technique where complete resection is balanced with functional or cosmetic outcome. Thus, survival depends on performing a radical resection balanced by conservative resection to preserve functional outcome; however, positive functional outcomes without successful oncologic outcomes are meaningless.

There are other clinical trial endpoints that could be considered as secondary endpoints. These include quality of life, retention of normal tissue, preservation of normal tissue function (i.e., nerves, ureters, lymphatics, vasculature), reduction of operative time and associated operating room costs, reduction in morbidities and complications related to prolonged general anesthesia, change in rate of positive margins, and reduction in the need for salvage surgery or adjuvant therapy when using the technique.

\section{CONCLUSION}

The field of optical imaging for surgical guidance is rapidly expanding with the introduction of new agents and hardware that will transition into the marketplace over the next $5 \mathrm{y}$. Submission of an IND to investigate safety, molecular targeting, and timing of surgery is the first step toward successful clinical implementation. As contrast-based optical imaging techniques are introduced into patients, the primary endpoints of initial clinical trials should demonstrate safety and that fluorescence imaging could be used to clearly delineate normal from tumor tissues. Ongoing interactions with the FDA are necessary to determine the regulatory pathway for IND submission and eventual clinical approval.

\section{DISCLOSURE}

The costs of publication of this article were defrayed in part by the payment of page charges. Therefore, and solely to indicate this fact, this article is hereby marked "advertisement" in accordance with 18 USC section 1734. No potential conflict of interest relevant to this article was reported.

\section{ACKNOWLEDGMENTS}

This document is the result of a consensus meeting held by the International Society of Image Guided Surgery (ISIGS) on February
6, 2015, at Miami, Florida, and reflects a synthesis of the participants' opinions and concepts for the clinical translation and application of optical imaging in surgery.

\section{REFERENCES}

1. Chambers KJ, Kraft S, Emerick K. Evaluation of frozen section margins in highrisk cutaneous squamous cell carcinomas of the head and neck. Laryngoscope. 2015;125:636-639.

2. Zarbo RJ, Hoffman GG, Howanitz PJ. Interinstitutional comparison of frozensection consultation: a College of American Pathologists Q-Probe study of 79,647 consultations in 297 North American institutions. Arch Pathol Lab Med. 1991;115:1187-1194.

3. Stummer W, Novotny A, Stepp H, Goetz C, Bise K, Reulen HJ. Fluorescenceguided resection of glioblastoma multiforme by using 5 -aminolevulinic acidinduced porphyrins: a prospective study in 52 consecutive patients. J Neurosurg. 2000;93:1003-1013.

4. Stummer W, Pichlmeier U, Meinel T, et al. Fluorescence-guided surgery with 5-aminolevulinic acid for resection of malignant glioma: a randomised controlled multicentre phase III trial. Lancet Oncol. 2006;7:392-401.

5. Day KE, Beck LN, Deep NL, Kovar J, Zinn KR, Rosenthal EL. Fluorescently labeled therapeutic antibodies for detection of microscopic melanoma. Laryngoscope. 2013;123:2681-2689.

6. Rosenthal EL, Kulbersh BD, Duncan RD, et al. In vivo detection of head and neck cancer orthotopic xenografts by immunofluorescence. Laryngoscope. 2006;116: 1636-1641.

7. van Dam GM, Themelis G, Crane LM, et al. Intraoperative tumor-specific fluorescence imaging in ovarian cancer by folate receptor- $\alpha$ targeting: first in-human results. Nat Med. 2011;17:1315-1319.

8. Kulbersh BD, Duncan RD, Magnuson JS, Skipper JB, Zinn K, Rosenthal EL. Sensitivity and specificity of fluorescent immunoguided neoplasm detection in head and neck cancer xenografts. Arch Otolaryngol Head Neck Surg. 2007;133: 511-515.

9. Bertino JS Jr, Greenberg HE, Reed MD. American College of Clinical Pharmacology. American College of Clinical Pharmacology position statement on the use of microdosing in the drug development process. J Clin Pharmacol. 2007; 47:418-422.

10. Sarapa N. Exploratory IND: a new regulatory strategy for early clinical drug development in the United States. Ernst Schering Res Found Workshop. 2007;59: 151-163.

11. Medical imaging and drug development. U.S. Food and Drug Administration website. http://www.fda.gov/Drugs/DevelopmentApprovalProcess/DevelopmentResources/ucm092895.htm. Updated December 4, 2014. Accessed October 7, 2015 .

12. Guidance for industry, investigators, and reviewers exploratory IND studies. U.S. Food and Drug Administration website. http://www.fda.gov/downloads/drugs/ guidancecomplianceregulatoryinformation/guidances/ucm078933.pdf. Accessed October 7, 2015.

13. Korb ML, Hartman YE, Kovar J, Zinn KR, Bland KI, Rosenthal EL. Use of monoclonal antibody-IRDye $800 \mathrm{CW}$ bioconjugates in the resection of breast cancer. J Surg Res. 2014;188:119-128.

14. Warram JM, de Boer E, Sorace AG, et al. Antibody-based imaging strategies for cancer. Cancer Metastasis Rev. 2014;33:809-822.

15. Cohen R, Stammes MA, de Roos IH, Stigter-van Walsum M, Visser GW, van Dongen GA. Inert coupling of IRDye800CW to monoclonal antibodies for clinical optical imaging of tumor targets. EJNMMI Res. 2011;1:31-43.

16. PreMarket approval (PMA). U.S. Food and Drug Administration website. http://www.accessdata.fda.gov/scripts/cdrh/cfdocs/cfPMA/pma.cfm. Updated October 5, 2015. Accessed October 7, 2015.

17. $510(\mathrm{k})$ premarket submission. U.S. Food and Drug Administration website. http://www.accessdata.fda.gov/scripts/cdrh/cfdocs/cfpmn/pmnsimplesearch.cfm. Updated October 5, 2015. Accessed October 7, 2015.

18. Basaran D, Salman MC, Boyraz G, et al. Accuracy of intraoperative frozen section in the evaluation of patients with adnexal mass: retrospective analysis of 748 cases with multivariate regression analysis. Pathol Oncol Res. 2015;21:113-118.

19. Novita G, Filassi JR, Ruiz CA, et al. Evaluation of frozen-section analysis of surgical margins in the treatment of breast cancer. Eur J Gynaecol Oncol. 2012;33: 498-501.

20. Zinn KR, Korb M, Samuel S, et al. IND-directed safety and biodistribution study of intravenously injected cetuximab-IRDye800 in cynomolgus macaques. Mol Imaging Biol. 2015;17:49-57. 